\title{
Modified Gravity Explains Dark Matter?
}

\author{
Taishi Katsuragawa* and Shinya Matsuzaki \\ Department of Physics, Nagoya University \\ 464-8602, Nagoya, Japan \\ E-mail: taishi@th.phys.nagoya-u.ac.jp \\ synya@hken.phys.nagoya-u.ac.jp
}

The modification of gravity leads to the emergence of new degrees of freedom described by the dynamical field, which can play a role of the dark energy and cause the late-time accelerated expansion of the Universe. Besides the dark energy problem, it has been suggested that the new particle derived from the modified gravity can be a dark matter candidate. Recently, we investigated the above scenario in the $F(R)$ gravity. The $F(R)$ gravity includes the extra scalar field in addition to the graviton. Since this scalar field originates from the gravitational theory, it has very weak interactions with the standard model particles. Moreover, the scalar field becomes heavy in the high-density region because of the chameleon mechanism. These two properties suggest that the scalar field can be the cold dark matter. We discuss the nature of this scalar field in the framework of particle physics and evaluate the lifetime of the new scalar particle.

The 3rd International Symposium on "Quest for the Origin of Particles and the Universe" 5-7 January 2017

Nagoya University, Japan

* Speaker. 


\section{Introduction}

The modified gravity theory has been intensively investigated so far to study the dark energy (DE). The modification of gravity brings a new degree of freedom, which can mimic the role of the cosmological constant, so that one can explain the late-time cosmic acceleration without introducing the ad hoc cosmological constant. In addition to the DE problem, it has been suggested that a new particle derived from the modification of gravity can be a dark matter (DM) candidate [1]. In this scenario, one might be able to predict the ratio of the DE to the DM with respect to the energy density today. We study the DM candidate derived from the $F(R)$ gravity.

\section{2. $F(R)$ gravity and the Weyl transformation}

The action of $F(R)$ gravity defined as follows:

$$
S=\frac{1}{2 \kappa^{2}} \int d^{4} x \sqrt{-g} F(R)+S_{\text {Matter }},
$$

where $\kappa^{2}=8 \pi G=1 / M_{\mathrm{pl}}^{2}$ and $M_{\mathrm{pl}}$ is the reduced Planck mass. $F(R)$ is a function of the Ricci scalar $R$ : e.g. $F(R)=R$ in general relativity. The matter action $S_{\text {Matter }}$ is defined as $S_{\text {Matter }}=$ $\int d^{4} x \sqrt{-g} \mathscr{L}_{\text {Matter }}\left(g^{\mu v}, \Psi\right)$ where $\mathscr{L}_{\text {Matter }}$ is the matter Lagrangian density and $\Psi$ denotes the matter fields.

Next, we transform the metric as $g_{\mu \nu} \rightarrow \tilde{g}_{\mu v}=\mathrm{e}^{2 \sqrt{1 / 6} \kappa \varphi} g_{\mu \nu}$. This transformation is called the Weyl transformation. Under the Weyl transformation with a choice $\mathrm{e}^{2 \sqrt{1 / 6} \kappa \varphi} \equiv F_{R}(R)$, the action (2.1) is transformed into the following form:

$$
\begin{aligned}
& S=\frac{1}{2 \kappa^{2}} \int d^{4} x \sqrt{-\tilde{g}} \tilde{R} \\
& \quad+\int d^{4} x \sqrt{-\tilde{g}}\left[-\frac{1}{2} \tilde{g}^{\mu v}\left(\partial_{\mu} \varphi\right)\left(\partial_{v} \varphi\right)-\frac{1}{2 \kappa^{2}} \frac{F_{R}(R(\varphi)) R(\varphi)-F(R(\varphi))}{F_{R}^{2}(R(\varphi))}\right]+S_{\text {Matter }}
\end{aligned}
$$

Here, $F_{R}(R)=\partial_{R} F(R)$ for our convention. The gravitational theory described as in the action 2.2 is called the scalar-tensor theory: the scalar field $\varphi(x)$ acts as the gravitational force besides the tensor field $g_{\mu \nu}$.

Finally, we consider the effect of the scalar field to the matter sector. According to the Weyl transformation, the matter sector in the action (2.2) is given by the following form:

$$
S_{\text {Matter }}=\int d^{4} x \sqrt{-\tilde{g}} \mathrm{e}^{-4 \sqrt{1 / 6} \kappa \varphi} \mathscr{L}_{\text {Matter }}\left(g^{\mu \nu}=\mathrm{e}^{2 \sqrt{1 / 6} \kappa \varphi} \tilde{g}^{\mu v}, \Psi\right) .
$$

One finds that the dilatonic coupling between the scalar field $\varphi(x)$ to the matter fields $\Psi(x)$ shows up in (2.3) after the Weyl transformation. Hereafter, we call this scalar field as scalaron.

\section{Chameleon Mechanism}

Constraints from the violation of the equivalence principle in the Solar System often exclude modifications of gravity although the modifications are required for the DE on the cosmological 
scale. Therefore, we need to suppress the fifth force mediated by the new degree of freedom only in the smaller scale. Viable models of $F(R)$ gravity have a chameleon mechanism to screen the fifth force mediated by the scalaron and avoid the constraint.

We now consider the equation of motion for the scalaron field. By variation of 2.2] with respect to the scalaron field $\varphi$, we obtain the equation of motion of the scalaron:

$$
\begin{aligned}
\tilde{\square} \varphi & =V_{\text {eff }}^{\prime}(\varphi), \\
V_{\text {eff }}^{\prime}(\varphi) & \equiv V^{\prime}(\varphi)+\frac{\kappa}{\sqrt{6}} \mathrm{e}^{-4 \sqrt{1 / 6} \kappa \varphi} T_{\mu}^{\mu} .
\end{aligned}
$$

We find that the scalaron couples to the trace of the energy-momentum tensor in the equation of motion.

To see how the chameleon mechanism works, we consider the Starobinsky model [2] for example. The action of the Starobinsky model is defined as

$$
F(R)=R-\beta R_{c}\left[1-\left(1+\frac{R^{2}}{R_{c}^{2}}\right)^{-n}\right]
$$

with constants $n, \beta$, and $R_{c}>0 . R_{c}$ is constant curvature in the Starobinsky model, which is comparable to the cosmological constant $R_{c} \sim \Lambda$. Moreover, we consider the non-relativistic perfect fluid with the constant energy density for simplicity. The trace of energy-momentum tensor is then expressed as $T_{\mu}^{\mu}=g^{\mu v} T_{\mu \nu}=-\rho$. In the large curvature limit $R / R_{c} \gg 1$, we obtain the expression of the scalaron mass

$$
m_{\varphi}^{2} \approx \frac{R_{c}}{6 n(2 n+1) \beta}\left(\frac{\kappa^{2} \rho}{R_{c}}\right)^{2(n+1)} .
$$

Therefore, if $\rho$ is larger, the scalaron becomes heavier: in the bulk of the Universe, where the energy density is very small, the scalaron can be very light and produce the effective cosmological constant. On the other hand, in or around the massive objects, the Solar System or the Earth, the scalaron becomes heavy. Then, the Compton wavelength becomes short, and the scalaron is screened.

\section{Scalaron as Dark matter}

We recall the properties of the scalaron: (1) after the Weyl transformation, dilatonic interactions between the scalaron and the standard model (SM) particles appear, which is suppressed by the Planck mass $\kappa=1 / M_{\mathrm{pl}}$; (2) the scalaron mass is very large in the large curvature regime because of the chameleon mechanism. These two characteristic properties of the scalaron imply that a massive field weakly coupled with SM particles emerges. Therefore, it suggests that the scalaron could be a cold DM.

It is necessary to determine the form and magnitude of couplings between the scalaron and the SM particles described by the action 2.3. To take into account the chameleon mechanism, we consider the large curvature limit $R \gg R_{c}$, corresponding to the weak coupling limit $|\kappa \varphi| \ll 1$. In this limit, we can expand the dilatonic coupling $\mathrm{e}^{\kappa \varphi}$. 
In case of a massless field, the coupling with the scalaron does not appear because of the classical conformal invariance. For a massless vector field $A_{\mu}(x)$, one finds that the action is invariant under the Weyl transformation,

$$
S_{\text {MasslessVector }}=\int d^{4} x \sqrt{-\tilde{g}}\left[-\frac{1}{4} \tilde{g}^{\alpha \mu} \tilde{g}^{\beta v} F_{\alpha \beta} F_{\mu v}\right],
$$

where $F_{\mu \nu}=\partial_{\mu} A_{\nu}-\partial_{\nu} A_{\mu}$. For a massless fermion field $\psi(x)$, the action is transformed as

$$
S_{\text {MasslessFermion }}=\int d^{4} x \sqrt{-\tilde{g}}\left[\mathrm{e}^{-3 \sqrt{1 / 6} \kappa \varphi} i \bar{\psi} \tilde{\gamma}^{\mu} \tilde{\nabla}_{\mu} \psi-\frac{3 i}{2} \sqrt{\frac{1}{6}} \kappa \mathrm{e}^{-3 \sqrt{1 / 6} \kappa \varphi}\left(\partial_{\mu} \varphi\right) \bar{\psi} \tilde{\gamma}^{\mu} \psi\right] \text {. }
$$

Although the couplings between the scalaron and fermion seem to remain, one may transform the action (4.2) into the canonical form by redefining the fermion field $\psi \rightarrow \psi^{\prime}=\mathrm{e}^{-3 / 2 \sqrt{1 / 6} \kappa \varphi} \psi$,

$$
S_{\text {MasslessFermion }}=\int d^{4} x \sqrt{-\tilde{g}} i \bar{\psi}^{\prime} \tilde{\gamma}^{\mu} \tilde{\nabla}_{\mu} \psi^{\prime} .
$$

Thus, one can eliminate the scalaron coupling by the field redefinition in classical dynamics.

The scalaron disappears from the action but would be transferred to the path integral measure because the field redefinition involves the scalaron field. The modified path integral measure induces the scale anomaly, then, the couplings with massless vector fields with the gauge coupling constant $g$ show up

$$
\mathscr{L}_{\text {MasslessVector }}=-\frac{3 g^{2}}{4(4 \pi)^{2}}\left(\frac{3}{2} \sqrt{\frac{1}{6}} \kappa \varphi\right) \operatorname{tr}\left[F_{\mu \nu}^{2}\right]+\mathscr{O}\left(\kappa^{2} \varphi^{2}\right) .
$$

In case of massive fields, one finds the coupling through the mass terms. So, the coupling between the scalaron and massive vector field $A(x)$ is given by

$$
\mathscr{L}_{\text {MassiveVector }}\left(\tilde{g}^{\mu v}, A_{\mu}, \varphi\right)=\frac{2 \kappa \varphi}{\sqrt{6}} \cdot \frac{1}{2} m_{V}^{2} \tilde{g}^{\mu v} A_{\mu} A_{v}+\mathscr{O}\left(\kappa^{2} \varphi^{2}\right)
$$

For the massive fermion field $\psi(x)$, after the canonical normalization $\left(\psi^{\prime}=\mathrm{e}^{-3 / 2 \sqrt{1 / 6} \kappa \varphi} \psi\right)$, we find

$$
\mathscr{L}_{\text {MassiveFermion }}\left(\psi^{\prime}, \varphi\right)=\frac{\kappa \varphi}{\sqrt{6}} \cdot m_{F} \bar{\psi}^{\prime} \psi^{\prime}+\mathscr{O}\left(\kappa^{2} \varphi^{2}\right)
$$

\section{An effective model and lifetime of scalaron particle}

We first expand the scalaron field $\varphi$ around the background solution $\varphi=\varphi_{\min }: \varphi=\hat{\varphi}+\varphi_{\min }$, and treat the fluctuation $\hat{\varphi}$ as a particle. As we have seen, the scalaron potential changes through the chameleon mechanism according to the trace of the energy-momentum tensor. In order to take the chameleon mechanism into account properly, we need to specify the energy-momentum tensor. In this work, we assume that the bulk which consists of the SM particles is described by the perfect fluid, and the energy density $\rho$ is namely given as $\rho_{\mathrm{EW}} \sim(100 \mathrm{GeV})^{4}$. Because $\left|\kappa \varphi_{\text {min }}\right| \ll 1$, the scalaron coupling to the SM is approximately given by

$$
S_{\text {Matter }} \approx \int d^{4} x \sqrt{-\tilde{g}} \mathrm{e}^{-4 \sqrt{1 / 6} \kappa \hat{\varphi}} \mathscr{L}_{\mathrm{SM}}\left(\mathrm{e}^{2 \sqrt{1 / 6} \kappa \hat{\varphi}} \tilde{g}^{\mu \nu}, \Psi\right),
$$


so that we utilize the result in the previous section just by replacing $\varphi \rightarrow \hat{\varphi}$. Note that the environment dependence still implicitly remains in the scalaron mass.

For the scalaron to be a DM candidate, the scalaron lifetime has to be longer than the age of the universe. Studying the all decay channels to the SM particles, we find the upper limit of the scalaron mass [3]:

$$
m_{\varphi} \lesssim 0.23 \mathrm{GeV}
$$

Finally, we convert the mass bound (5.2) into the constraint on the $F(R)$ function. For the Starobinsky model with $\beta R_{c}=2 \Lambda$, the scalaron mass is given by substituting $\rho=\rho_{\mathrm{EW}}$ into Eq. (3.3). Then, we find $\beta \lesssim 10^{-69}$ for $n=1$, and $\beta \lesssim 10^{-59}$ for $n=4$.

\section{Summary and Discussion}

We have studied the scalaron field in the $F(R)$ gravity from the viewpoint of particle physics. We have assumed that the scalaron is a DM candidate and evaluated its lifetime from the decay to the SM particles. We finally have placed the constraint on the scalaron mass.

We have obtained the constraint on the parameter $\beta$ in the Starobinsky model and found that $\beta$ should be extremely small for the scalaron to be a DM although it is desired to be $\mathscr{O}(1)$ for the modified gravity to be a solution for the DE problem. This is because that the chameleon mechanism is designed to make the scalaron massive in the high-density region to avoid the Solar System constraint. The scalaron, however, should not be too heavy because we expect that the scalaron plays the role of a DM candidate in our scenario. Thus, the chameleon mechanism is the origin of discrepancy although it is one of the essences in our scenario.

However, we are still left with open questions to avoid the incompatibility because some assumptions were made to derive the constraint on the parameter $\beta$. In order to resolve the incompatibility, we need to consider new methodology or effective models. Besides, we will also study and improve the methods to study the thermal history, relic abundance, and prediction for direct detection of the scalaron.

\section{Acknowledgments}

This work is supported by the Grant-in-Aid for JSPS Fellows \#15J06973 (T.K.) and by the JSPS Grant-in-Aid for Young Scientists (B) \#15K17645 (S.M.).

\section{References}

[1] S. Nojiri and S. D. Odintsov, Dark energy, inflation and dark matter from modified $F(R)$ gravity, TSPU Bulletin N 8(110), 7 (2011) [arXiv:0807.0685 [hep-th] ].

[2] A. A. Starobinsky, Disappearing cosmological constant in $f(R)$ gravity, JETP Lett. 86, 157 (2007) [arXiv:0706.2041 [astro-ph]].

[3] T. Katsuragawa and S. Matsuzaki, Dark matter in modified gravity?, Phys. Rev. D 95, no. 4, 044040 (2017) [arXiv:1610.01016 [gr-qc]]. 